\title{
Variaciones en la flora apícola de una región de la Depresión del Salado (Buenos Aires, Argentina) referidas a cambios ambientales asociados con humedales. Implicancia económica
}

\author{
Ana Clara Dedomenici ${ }^{1}$, Domingo Leveratto ${ }^{2}$, José Ringuelet ${ }^{1}$ y Lilian Passarelli ${ }^{1}$
}

Resumen: Dedomenici, A. C.; Leveratto, D.; Ringuelet, J. \& Passarelli, L. 2011. Variaciones en la flora apícola de una región de la Depresión del Salado (Buenos Aires, Argentina) referidas a cambios ambientales asociados con humedales. Implicancia económica. Bot. Complut. 35: 141-145.

Se realizó el estudio del polen presente en las mieles de un colmenar ubicado en la zona de la Depresión del Salado, Buenos Aires, Argentina, con el objetivo de observar la flora utilizada por Apis melifera en un ambiente que ha sido afectado por periódicas inundaciones que modificaron la vegetación; realizando comparaciones con datos preexistentes. Se identificaron 31 tipos polínicos pertenecientes a 15 familias botánicas. Todas las muestras resultaron ser monoflorales de Lotus tenuis con porcentajes altos, mayores al 50\%. Los resultados muestran una variación importante respecto de datos anteriores en los recursos utilizados por la abeja doméstica en esta zona. Se realiza un nuevo aporte al conocimiento sobre el origen floral de las mieles de la región que incidirá en su valor comercial.

Palabras clave: polen, mieles, Lotus, producción apícola.

Abstract: Dedomenici, A. C.; Leveratto, D.; Ringuelet, J. \& Passarelli, L. 2011. Changes in bee flora of a region of the Depresión del Salado (Buenos Aires, Argentina) concerning environmental changes associated with wetlands. Economic implication. Bot. Complut. 35: 141-145.

A study of the pollen present in honey from an apiary located in the area of the Depresion del Salado, Buenos Aires, Argentina, in order to observe the plants used by Apis mellifera L. in an environment that has been affected by periodic floods that changed the vegetation, and making comparisons with existing data. Thirty one pollen types belonging to fifteen botanical families were identified. All samples were monofloral of Lotus tenuis with high percentages, above 50\%. The results show significant variation respect to previous data on resources used by the honeybee in this area. It makes a new contribution to knowledge of the floral origin of honey from the region that will affect their commercial value.

Key words: pollen, honey, Lotus, bee production.

\section{INTRODUCCION}

Durante los últimos años, la denominación de origen y el control de calidad de los productos son los principales requerimientos del Mercado Internacional de la Miel. Por esta razón conocer y certificar el origen floral constituye un importante aporte para la comercialización de los mismos. Por otra parte, los análisis melisopalinológicos son muy valiosos como estudios florísticos pues permiten conocer la vegetación circundante al apiario y su variación estacional y anual. La zona perteneciente a la Depresión del Salado presenta distintas comunidades, específicamente pertenece a una región de pastizales sub-húmedos
(Apostolo 2005, Soriano 1991). Durante los últimos 20 años esta zona de la Pampa Deprimida de la provincia de Buenos Aires ha sido afectada en gran parte por inundaciones periódicas, siendo una zona anegable en la que la vegetación ha cambiado de acuerdo con estas variaciones. Se emprende por lo tanto el estudio del polen presente en las mieles de un colmenar ubicado en la zona de la Depresión del Salado en el límite entre Chascomús y Magadalena con el objetivo de observar los recursos utilizados por Apis melifera L. durante los meses de cosecha y realizar así, un nuevo aporte al conocimiento de los recursos nectaríferos de la región, de acuerdo con la variación ambiental producida por las inundaciones en esta zona.

\footnotetext{
${ }^{1}$ Laboratorio de Estudios de Anatomía Vegetal Evolutiva y Sistemática (LEAVES), Facultad de Ciencias Naturales y Museo de La Plata, 64 entre 120 y diagonal 113, B1904 DZB, La Plata, Argentina. anadedomenici@hotmail.com; Impassarelli@yahoo.com.ar

${ }^{2}$ Cátedra de Producción Animal, Facultad de Ciencias Agrarias y Forestales, Universidad Nacional de La Plata, Calle 60 y 119, La Plata, Argentina.abejas@uolsinectis.com.ar

Recibido: 25 enero 2011. Aceptado: 3 marzo 2011
} 


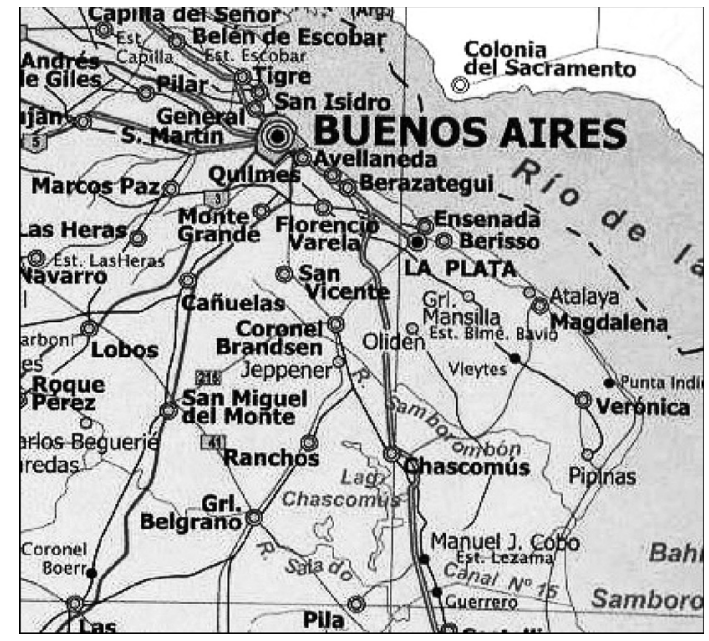

Fig. 1- Mapa de la zona de estudio.

\section{MATERIALES Y METODOS}

Las muestras provienen de un apiario de la zona de la depresión del Salado, ubicado a 5 metros del río Samborombón, en el partido de Magdalena, provincia de Buenos Aires (Fig. 1). Se colectaron 10 muestras durante los meses de enero, febrero y marzo de 2006. La periodicidad de muestreo fue cada 10 días y todas las muestras corresponden a una cosecha diferente. En los años 2008 y 2009 se realizaron nuevos análisis con el objeto de observar si se mantenía el origen botánico de Lotus. Para el análisis cualitativo las mieles fueron tratadas según las técnicas de Louveaux et al. (1978). El recuento de granos se realizó hasta la estabilización de los porcentajes (Vergeron 1964). Los taxones fueron observados con MO (microscopio óptico) y determinados mediante su comparación con preparados de referencia pertenecientes al Laboratorio de Melisopalinología del Ministerio de Asuntos Agrarios de la provincia de Buenos Aires y del LEAVES de la Facultad de Ciencias Naturales y Museo de la Universidad Nacional de La Plata. Fueron utilizados además atlas palinológicos y trabajos referidos a la zona de estudio (Markgraf et al. 1978). En forma complementaria se realizaron observaciones de la flora en el campo, durante los meses muestreados. Con los resultados de los análisis de las mieles se confeccionó una tabla de clases de frecuencia relativa considerando los siguientes valores: polen dominante, D: con frecuencia mayor al $45 \%$ del polen total; polen secundario, $\mathbf{S}$ : entre el 15 y $45 \%$ del total; polen de menor importancia, M: entre el 3-14\% y polen en trazas, $t$ : inferior al $3 \%$ del total (Louveaux et al. 1978).

\section{RESULTADOS}

Los resultados están resumidos en la Tabla 1. Se identificaron 31 tipos polínicos pertenecientes a15 familias, siendo las Fabaceae y las Asteraceae las más representadas. Todas las muestras resultaron ser monofloras de $L o$ tus tenuis Waldst. et Kit., con porcentajes altos, mayores al $50 \%$. Es importante destacar que los valores de L. tenuis registrados son mucho más elevados que los porcentajes de otras especies presentes en las muestras (Fig. 2).

Algunos taxones como Carduus sp. y Eucalyptus sp. están representados en todas las muestras. Otros como Apiaceae, Brassica sp., Taraxacum officinale Weber y Trifolium sp., tienen un aporte casi continuo, encontrándose en floración durante la mayor parte del período observado. Sin embargo, en el caso de Apiaceae aparece como recurso secundario en enero y disminuye su contribución a valores despreciables en los siguientes meses. También en enero es importante el aporte de Mentha sp. taxón que disminuye en los meses siguientes. Durante febrero Carduus sp. y Lotus tenuis aumentan considerablemente sus porcentajes en la miel, y en el mismo período disminuye la presencia de polen de Eucalyptus sp. (Fig. 2). Durante marzo Carduus sp., Melilotus sp. y Taraxacum sp. junto con Lotus tenuis son los recursos más importantes utilizados por Apis mellifera aunque los tres primeros aparecen significativamente en menor proporción.

Cuando se comparan los valores obtenidos con los estudios previos realizados en la región, (Basualdo et al 2006, Tellería 1992) comprobamos que algunos taxones como Brassicaceae, Carduus sp., Centaurea sp., Cichorium sp., Echium plantagineum, Mentha sp., Trifolium pratense y Trifolium repens siguen presentes. Sin embargo al comparar las frecuencias con Lotus tenuis observamos que se registró un aumento significativo en el aporte de este taxón en los últimos 15 años debido a períodos de inundaciones. Según Tellería (1992) Lotus tenuis presentaba en la mayoría de las muestras una frecuencia entre el 3 y el 14\%, siendo considerado como polen de menor importancia, valores muy distantes al $50 \%$ observado en este trabajo.

\section{DISCUSIÓNY CONCLUSIONES}

Los resultados obtenidos muestran que todas las mieles son monofloras de Lotus tenuis y que los valores de frecuencia son mayores al 50\%. Ambos resultados constituyen datos económicos importantes que deberían ser aprovechados por los apicultores, ya que según la legislación vigente (SAGPyA 1995) se determinaría a esta miel como monoflora de tréboles desde un $45 \%$ de frecuencia y la constancia en la exclusividad de mieles de Lotus tenuis en los tres meses habituales de cosecha aseguraría la 
Tabla 1

Tabla 1. Clases de frecuencia de polen en las distintas muestras de miel. Todos los porcentajes marcados para Lotus tenuis corresponden a la clase de frecuencia: D

\begin{tabular}{lcccccccccc}
\hline \multirow{2}{*}{ Taxón } & Ene & Ene & Ene & Feb & Feb & Feb & Feb & Mar & Mar & Mar \\
& M1 & M2 & M3 & M4 & M5 & M6 & M7 & M8 & M9 & M10 \\
\hline Apiaceale & S & S & M & M & M & M & t & \multirow{2}{*}{ t } & & t
\end{tabular}

\section{Asteraceae}

Carduus sp.

Centaurea sp.

Helianthus annuus $\mathrm{L}$.

Tipo Taraxacum sp.

Tipo Bacharis sp.

Tipo Bidens sp.

Otras

$\begin{array}{cccccccccc}t & t & M & M & M & S & t & M & t & M \\ t & t & t & t & t & & & t & & \\ P & & & & & & & & & t \\ & t & t & t & t & t & t & t & t & M \\ & & t & t & & & t & t & & \\ & t & & & & & & t & & \end{array}$

\section{Borraginaceae}

Echium sp.

Heliotropum sp.

\section{Brassicaceae}

Brassica sp.

\section{Convolvulaceae}

\section{Fabaceae}

Lotus tenuis

Melilotus sp.

Parkinsonia sp.

Trifolium sp.

Trifolium pratense $\mathrm{L}$.

tipo Melilotus sp.

Tipo Lathyrus sp.

Otras

\section{Gramineae}

Lamiaceae

Mentha sp.

Otras

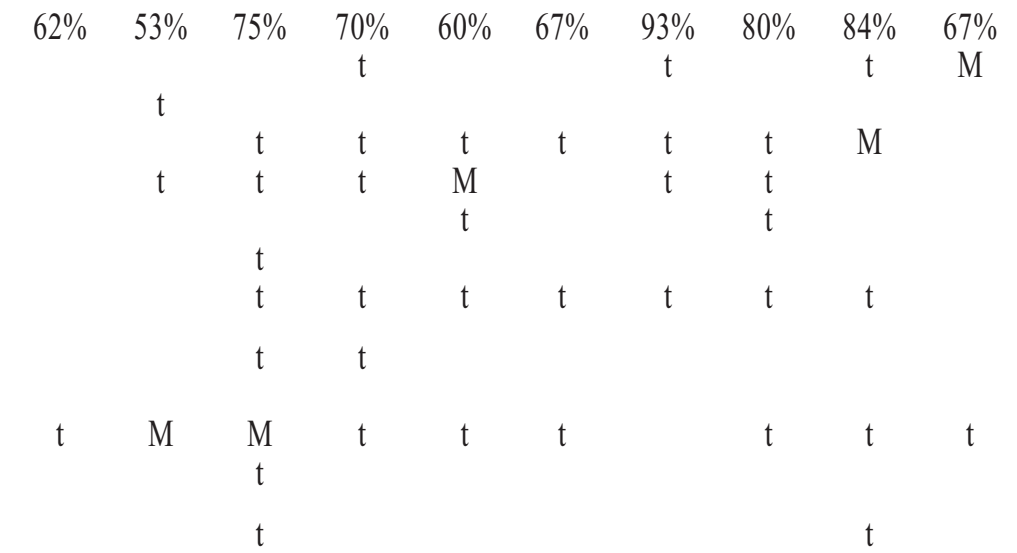

Malvaceae

Myrtaceae

Eucalyptus sp.

\section{Oleaceae}

Ligustrum sp.

Quenop-Amarantaceae

\section{Salicaceae}

\section{Urticaceae}

Urtica sp.

Verbenaceae

Otros

t

t

t

M

$t \quad t \quad t \quad t$




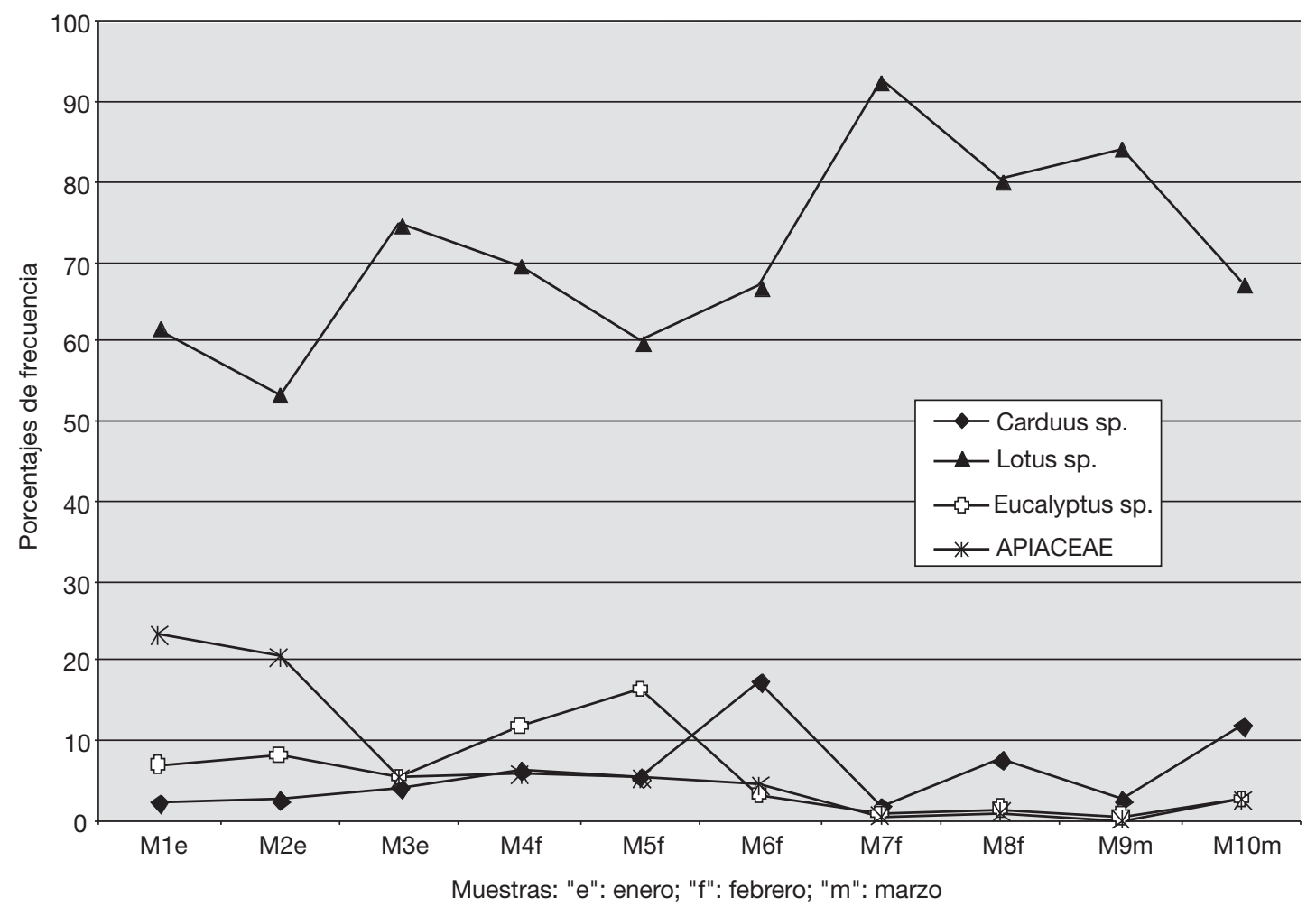

Fig. 2- Porcentajes del polen presente en las muestras de miel.

obtención de una producción monofloral, con valores comerciales significativamente mayores que aquellos de la miel a granel. La presencia de mieles monofloras de Lotus tenuis ha sido observada en otras zonas de la pradera húmeda por los autores con porcentajes similares al de este trabajo, constituyendo un potencial para el desarrollo de la región.

Algunos de los táxones identificados han sido mencionados en estudios previos (Basualdo et al 2006, Tellería 1992) sin embargo el aumento en el aporte de Lotus tenuis en los últimos años es significativo, con una gran diferencia entre las frecuencias de este taxón y el resto de los presentes en las muestras, algo no observado en los trabajos previos mencionados. Lotus tenuis es una especie naturalizada en Argentina, sus poblaciones se están extendiendo debido al cambio de las condiciones ambientales en la zona, con un importante aumento de la humedad edáfica, factor que ha contribuido a su expansión.

Secundariamente, el mayor aporte de néctar, es de otras especies pertenecientes a las familias Fabaceae, Asteraceae, Apiaceae, Myrtaceae y Lamiaceae, este dato concuerda con lo conocido hasta el momento para provincia de Buenos Aires, con una importante flora introducida y predominancia de taxones europeos en las mieles (Tellería 1988), contrariamente a lo observado para mieles de otras provincias argentinas que poseen con mayor frecuencia especies nativas (Caccavari \& Fagúndez 2004). Las familias mencionadas anteriormente están presentes durante toda la temporada de cosecha, desde enero a marzo, aunque el aporte varía notablemente en los distintos meses. Además, es claramente observable una disminución de la riqueza hacia el verano tardío (febrero-marzo). Dichas variaciones se ven reflejadas en la floración de la zona, es decir, los resultados obtenidos del análisis coinciden ampliamente con lo observado en el campo.

\section{AGRADECIMIENTOS}

Este trabajo ha sido realizado con el apoyo del Programa de Incentivos a Docentes Investigadores de la Facultad de Ciencias Naturales y Museo, Universidad Nacional de La Plata, Proyecto No $11 /$ N558. 


\section{BIBLIOGRAFÍA}

Apostolo, N. M. 2005. Caracteres anatómicos de la vegetación costera del Río Salado (Noroeste de la Provincia de Buenos Aires, Argentina. Bol. Soc. Argentina Bot. 40:215-227.

Basualdo, M.; Pereda, A. \& Bedascarrabure, E. 2006. Caracterización botánica y geográfica de mieles de la Cuenca del Salado, provincia de Buenos Aires, Argentina. RIA 35(1): 5-14.

CACCAVARI, M. \& FacúnDEZ, G. 2004. Alcances de la biodiversidad en mieles del Litoral Fluvial. Un enfoque agroecológico. INSUGEO. Miscelánea 12: 147-151.

Louveaux, J.; Maurizio, A. \& Vorwhol, G. 1978. Methods of Melissopalynology by International Comission for bee Botany of IUBS Bee World 59: 139-157.

Markgraf, V. \& D'Antoni, H. L. 1978. Pollen flora of Argentina modern spore and pollen types of Pteridophyta, Gymnospermae, and Angiospermae. University of Arizona Press.
Sagpya. 1995. Sistema de clasificación de la miel teniendo como base su origen botánico. Secretaría de Agricultura, Ganadería, Pesca y Alimentación de la República Argentina. Resolución 274/95. Boletín Oficial n 28268 (1): 2.

Soriano, A. 1991. Río de la Plata Grasslands. En: R. T. Coupland (Ed.), Natural Grasslands. Introduction and Western Hemisphere 19: 367-407. Elsevier, Amsterdam.

TelLería, M. C. 1988. Analyse pollinique des miels du nordouest de la province de Buenos Aires (République Argentine). Apidologie 19: 275-290.

TelLeRíA, M. C. 1992. Caracterización botánica y geográfica de las mieles de la provincia fitogeográfica Pampeana (República Argentina) I: Distrito Oriental. Darwiniana 31: 345-350.

VeRGERON, N. 1964. Intérpretation statstique des résultats en matière d'analyse pollinique des miels. Ann. Abeille 7: 349- 364. 